\title{
Ursell Operators in Statistical Physics III: thermodynamic properties of degenerate gases
}

\author{
P. Grüter (1), F. Laloë (1), A.E. Meyerovich (2) and W. Mullin (3) \\ (1) Laboratoire Kastler Brossel de l'ENS* \\ 24 rue Lhomond, F 75005 Paris, France \\ (2) Department of Physics, University of Rhode Island \\ Kingston RI 02881, USA \\ (3) Hasbrouck Laboratory, University of Massachusetts \\ Amherst Mass 01003, USA
}

\begin{abstract}
We study in more detail the properties of the generalized Beth Uhlenbeck formula obtained in a preceding article. This formula leads to a simple integral expression of the grand potential of any dilute system, where the interaction potential appears only through the matrix elements of the second order Ursell operator $U_{2}$. Our results remain valid for significant degree of degeneracy of the gas, but not when Bose Einstein (or BCS) condensation is reached, or even too close to this transition point. We apply them to the study of the thermodynamic properties of degenerate quantum gases: equation of state, magnetic susceptibility, effects of exchange between bound states and free particles, etc. We compare our predictions to those obtained within other approaches, especially the "pseudo potential" approximation, where the real potential is replaced by a potential with zero range (Dirac delta function). This comparison is conveniently made in terms of a temperature dependent quantity, the "Ursell length", which we define in the text. This length plays a role which is analogous to the scattering length for pseudopotentials, but it is temperature dependent and may include more physical effects than just binary collision effects; for instance, for fermions at very low temperatures, it may change sign or increase almost exponentially. As an illustration, numerical results for quantum hard spheres are given.
\end{abstract}

*Laboratoire associé au CNRS, UA 18, et à l'Université Pierre et Marie Curie 


\section{Introduction}

The use of quantum cluster expansions was introduced in 1938 by Kahn and Uhlenbeck [1], who generalized to quantum statistical mechanics the Ursell functions $U_{q}$ defined by this author in 1927 [2]. The major virtue of cluster expansions is that they provide directly density expansions for systems where the interaction potential is not a necessarily small perturbation; in fact, it may even diverge at short relative distances (hard cores for instance) while usual perturbations theories generate power series in the interaction potential, where each term becomes infinite for hard core potentials. Starting from a quantum cluster analysis, the Beth Uhlenbeck formula [3, 4, 5] gives an explicit expression of the first terms of a fugacity expansion (or virial expansion) for the grand potential of a quantum gas. The expression is valid for any potential, the latter being characterized by its phase shifts in a completely general way.

One should nevertheless keep in mind that the words "density expansion" have a double meaning in this context. In a dilute gas, there are actually two dimensionless parameters which characterize "diluteness": the product $n^{1 / 3} b$, where $n$ is the number density of the gas and $b$ is a length characterizing the potential range (diameter of hard cores for instance), and the product $n^{1 / 3} \lambda_{T}$, where $\lambda_{T}$ is the quantum thermal wavelength of the particles. The former parameter is small if, classically, a snapshot of the system shows particles among which almost all are moving freely, while the few that interact are engaged in binary collisions only; the latter, purely quantum in nature, is sometimes called the quantum degeneracy parameter, and remains small provided there is little overlap of the quantum wave packets. The validity of the Beth Uhlenbeck formula, as all fugacity expansions, therefore requires two independent parameters to be small.

In a previous article [6], we discuss one method which conveniently treats the two parameters separately, and allows one to include the effects of statistics by exact summations while limiting the expansion to the lowest orders in $n^{1 / 3} b$. The technique is based on the use of Ursell operators $U_{q}$ generalizing the Ursell functions (for a system of distinguishable particles), coupled with the exact calculation of the effect of exchange cycles $C_{l}$ of arbitrary length $l$; for short we call it the technique of $\mathrm{U}-\mathrm{C}$ diagrams. The result is another expression of the grand potential, which is no longer a fugacity expansion since it includes a summation over all sizes of exchange cycles so that statistical effects are included to all orders. Truncating the expansion to its first terms (lowest $q$ values and/or low order in a given $U_{q}$ ) gives results which remain valid for "dilute degenerate systems" [7], that is for all systems where the potential range is sufficiently small, but where the degeneracy of the system may become significant円. In this article we will

\footnotetext{
${ }^{1}$ For bosons, Bose Einstein condensation is excluded since it requires a summation over an
} 
start from an expression of the grand potential which is limited to the first order correction in the second Ursell operator $U_{2}$, obtained in [6] as a trace over two particles of a product of operators. We reduce the trace to an explicit integral where the effects of the interactions are contained in a simple matrix element. The range of validity of our result is actually similar to that of the calculations based on the use of pseudopotentials [8], another approach where the final results automatically include the summation of an infinite perturbation series in terms of the initial potential. The two methods are comparable, but we think that the $\mathrm{U}-\mathrm{C}$ diagram method provides more general and more precise results, basically because it includes the short range correlations between the particles, and because all scattering channels with given angular momentum as well as their exact energy dependence are included instead of only one constant scattering length? Another point of comparison is the class of methods, for instance discussed in [10] or [11], where a renormalization procedure is used in order to obtain expansions in terms of the scattering $T$ matrix instead of the interaction potential itself $V$; in the calculations discussed in the present article, no renormalization of this kind is needed since, roughly speaking, it is already included in the $U_{q}$ 's, which are the building blocks of our method. Nevertheless, as we will see, our method is no longer valid when the gaseous system is brought too close to a phase transition (superfluid transition of single particles for bosons, of pairs for fermions).

We begin this article with a study of the expression of the grand potential, and show how it can be expressed as an expression that is similar to the well known Beth Uhlenbeck formula; actually it can be obtained from it by two simple substitutions. We then discuss the physics contained in this general result, as well as the changes introduced by the possible occurrence of bound states. In particular we consider the effects of exchange between bound and unbound particles, an effect which is not contained in the usual Beth Uhlenbeck formalism; this kind of exchange may play some role in clouds of laser cooled alkali atoms [12, 13, 14] for which the potential is sufficiently attractive to sustain a large number of bound states. In section 3 we apply these results to spin $1 / 2$ particles. Finally, in section 4, we discuss the appropriate quantities in terms of which one should describe the effects of the potential on the physical properties of the system, and introduce for this purpose the so called "Ursell length", which plays a role similar to the scattering length $a$. In the theoretical study of quantum gases, and as already mentioned, one frequently used method is to replace the real interaction potential between the particles by a "pseudopotential" that has no range (a Dirac delta function of the space variables), and to treat this potential to first perturbation order; in other words one ignores the distortion of the many-body wave functions at short relative distances and the associated effects of the inter particle corre-

infinite number of interaction terms, a question which we will study in a forthcoming article

${ }^{2}$ This does not mean that one could not improve the theory of pseudopotentials to include all phase shifts, since a general expression of the pseudopotential is given by Huang in [9], but to our knowledge this has not been done explicitly. 
lations. The justification of this approach is based on the physical expectation that, for a dilute gas, all the effects of the potential should be contained in the binary collision phase shifts associated with the potential, which can easily be reproduced to first order by a pseudopotential; meanwhile all detailed information on the behavior of the wave function at short relative distances can safely be discarded. Our formalism allows one to explicitly distinguish between short range effects ("in potential effects") and asymptotic effects (out of the potential), which naturally leads to a discussion of this ansatz. The interactions appear in terms of a matrix element of an Ursell operator, which depends on the potential but does not reduce to it; for fermions at low temperatures, the matrix elements contain physical effects which are not included in usual treatments of normal Fermi gases.

\section{The grand potential}

\section{$2.1 \quad$ Notation}

The basic object in terms of which most physical quantities will be written in this article is the second Ursell operator $U_{2}$, defined by:

$$
U_{2}(1,2)=\left[\mathrm{e}^{-\beta H_{2}(1,2)}-\mathrm{e}^{-\beta\left[H_{1}(1)+H_{1}(2)\right]}\right]
$$

where $H_{1}(1)$ and $H_{1}(2)$ are single particle hamiltonians, containing the kinetic energy of the particle and, if necessary, its coupling to an external potential, and where:

$$
H_{2}(1,2)=H_{1}(1)+H_{1}(2)+V_{\text {int }}(1,2)
$$

is the hamiltionan of two particles, including the mutual interaction potential $V_{\text {int }}(1,2)$. Depending of the context, it may be more convenient to use the symmetrized operator $U_{2}^{S, A}$ :

$$
U_{2}^{S, A}(1,2)=U_{2}(1,2) \frac{\left[1+\eta P_{e x}\right]}{2}=\frac{\left[1+\eta P_{e x}\right]}{2} U_{2}(1,2)
$$

where $P_{e x}$ is the exchange operator between particles 1 and 2 and $\eta$ has the value +1 for bosons, -1 for fermions. Moreover, the "interaction representation version" of either $U_{2}$ and $U_{2}^{S, A}$, obtained by multiplying these operators by

$e^{\beta H_{1}(1)} e^{\beta H_{1}(2)}$, will also be useful; we denote them with an additional bar over the operator, for instance:

$$
\bar{U}_{2}(1,2)=\mathrm{e}^{\beta H_{1}(1)} \mathrm{e}^{\beta H_{1}(2)} \mathrm{e}^{-\beta H_{2}(1,2)}-1
$$

This operator and its symmetrized version $\bar{U}_{2}^{S, A}(1,2)$ act only in the space of relative motion of the two particles; they have no action at all on the variables of the center of mass. 
Finally, if the particles are not submitted to an external potential $\left(H_{1}\right.$ contains only the kinetic energy), it is convenient to introduce the momentum $\mathbf{P}_{G}$ of the center of mass of the two particles as well as the hamiltonian of the relative motion:

$$
H_{r e l}=\frac{\mathbf{P}^{2}}{m}+V_{\text {int }}(\mathbf{R})
$$

( $m$ is the mass of the particles); then, $U_{2}(1,2)$ can be written in the form of a product:

$$
U_{2}(1,2)=\mathrm{e}^{-\beta\left(\mathbf{P}_{G}\right)^{2} / 4 m} \times\left[U_{2}(1,2)\right]_{r e l}
$$

with:

$$
\left[U_{2}(1,2)\right]_{r e l}=\left[\mathrm{e}^{-\beta H_{r e l}}-\mathrm{e}^{-\beta \frac{\mathbf{P}^{2}}{m}}\right]
$$

\subsection{Approximate expression of the thermodynamic po- tential}

We now start from relation (46) of [6] which gives the grand potential (multiplied by $-\beta$ ) in the form:

$$
\log Z=[\log Z]_{i g}+[\log Z]_{i n t}
$$

where $[\log Z]_{i g}$ is the well known value of $\log Z$ for the ideal gas:

$$
\begin{gathered}
{[\log Z]_{i g}=-\eta \operatorname{Tr}\left\{\log \left[1-\eta z \mathrm{e}^{-\beta H_{1}}\right]\right\}} \\
=\eta \operatorname{Tr}\{\log [1+\eta f]\}
\end{gathered}
$$

and where the correction introduced by the interactions is:

$$
[\log Z]_{i n t}=z^{2} \operatorname{Tr}_{1,2}\left\{U_{2}^{S, A}(1,2)[1+\eta f(1)][1+\eta f(2)]\right\}
$$

In these equations,

$$
z=\mathrm{e}^{\beta \mu}
$$

is the fugacity, $\beta=1 / k_{B} T$ the inverse temperature and $\mu$ the chemical potential, while $f$ is defined as the operator:

$$
f=\frac{z \mathrm{e}^{-\beta H_{1}}}{1-\eta z \mathrm{e}^{-\beta H_{1}}}
$$

Similar results can be found in the work of Lee and Yang, see formulas (II.8) and (II.23) of ref. [15]. As mentioned in the introduction, equation (10) gives the correction introduced by the interactions to the lowest order approximation in $U_{2}$; see [6] for a discussion of the higher order corrections. Using the definition of $\bar{U}_{2}^{S, A}$ :

$$
\bar{U}_{2}^{S, A}(1,2)=\mathrm{e}^{\beta H_{1}(1)} \mathrm{e}^{\beta H_{1}(2)} U_{2}^{S, A}(1,2)=\frac{1+\eta P_{e x}}{2}\left[\mathrm{e}^{\beta H_{1}(1)} \mathrm{e}^{\beta H_{1}(2)} \mathrm{e}^{-\beta H_{2}(1,2)}-1\right]
$$


as well as the relation $z \mathrm{e}^{-\beta H_{1}}[1+\eta f]=f$, we can rewrite (10) in the form:

$$
[\log Z]_{i n t}=\operatorname{Tr}_{1,2}\left\{\bar{U}_{2}^{S, A}(1,2) f(1) f(2)\right\}
$$

which expresses the correction as the average of the operator $\bar{U}_{2}^{S, A}(1,2)$ over unperturbed distributions functions $f$ 's.

\subsection{Spinless particles and rotational invariance}

For spinless particles, by making the trace in (14) explicit, we obtain:

$$
[\log Z]_{\text {int }}=-\frac{\lambda_{T}^{2} \mathcal{V}}{(2 \pi)^{6}} \int d^{3} k_{1} \int d^{3} k_{2} f\left(\mathbf{k}_{1}\right) f\left(\mathbf{k}_{2}\right) a_{U}^{S, A}\left(\left|\mathbf{k}_{1}-\mathbf{k}_{2}\right|\right)
$$

where $\mathcal{V}$ is the volume of the system, $\lambda_{T}$ the thermal wavelength:

$$
\lambda_{T}=\frac{h}{\sqrt{2 \pi m k_{B} T}}
$$

and where $a_{U}^{S, A}(k)$ is defined by:

$$
a_{U}^{S, A}(k)=-\frac{\mathcal{V}}{\lambda_{T}^{2}}<\mathbf{k}\left|\bar{U}_{2}^{S, A}\right| \mathbf{k}>=-\frac{\mathcal{V}}{\lambda_{T}^{2}} e^{\beta \hbar^{2} k^{2} / m}<\mathbf{k}\left|\left[U_{2}^{S, A}\right]_{r e l}\right| \mathbf{k}>
$$

Here:

$$
\mathbf{k}=\frac{\mathbf{k}_{1}-\mathbf{k}_{2}}{2}
$$

is the appropriate variable since $\bar{U}_{2}^{S, A}(1,2)$ does not have any action in the space of states associated to the center of mass of the two particles; $a_{U}^{S, A}(k)$ is a microscopic length, independent of $\mathcal{V}$ for large systems 3 , which we will call the "Ursell length" - see section 4.1 for a more detailed discussion and a justification of the numerical factors that we have introduced. The correction written in (15) is analogous to a first order energy correction due to binary interactions, while $a_{U}^{S, A}(k)$ plays the role of some effective interaction (within a numerical factor).

For instance, if we assume that we treat to first order in perturbation a pseudopotentiall of the form:

${ }^{3}$ This is true since $\bar{U}_{2}^{S, A}(1,2)$ has a microscopic range and since the factor $\mathcal{V}$ in $(17)$ makes up for the normalization factor of the plane waves that occur in the matrix element (note that all plane wave kets in our formulas are normalized in a finite volume; hence the absence of Dirac delta functions of momenta differences in (17).

${ }^{4}$ The most usual procedure is to treat this potential to first order only, since a naive treatment of higher orders may introduce inconsistencies. For instance, in three dimensions, it is possible to show that all phase shifts, and therefore the collision cross section, of a zero range potential such as (19), are exactly zero; on the other hand, they do not vanish to first order (in other words, the Born series for potentials containing a delta function is not convergent). For the same reason, for a potential such as (19), the Ursell operator $U_{2}$ vanishes exactly, while it does not if the potential is treated to first order.

For a more elaborate discussion of pseudopotentials going beyond (19) and including waves of higher angular momentum, see [9]. 


$$
V_{\text {eff }}(\mathbf{r})=\frac{4 \pi \hbar^{2} a}{m} \delta(\mathbf{r})
$$

where $a$ is a scattering length (or the diameter of hard cores), we easily obtain:

$$
<\mathbf{k}\left|\bar{U}_{2}^{S, A}\right| \mathbf{k}>\simeq-\frac{\lambda_{T}^{2}}{\mathcal{V}} a[1+\eta]
$$

so that (17) shows that, in this approximation, the Ursell length becomes independent of the wave number $k$. For bosons, the correction to the grand potential then becomes:

$$
[\log Z]_{i n t} \simeq-2 a \frac{\mathcal{V}}{\lambda_{T}} \times g_{3 / 2}(z)
$$

with the usual notation:

$$
g_{3 / 2}(z)=\frac{\lambda_{T}^{3}}{(2 \pi)^{3}} \int d^{3} k f(k ; z)
$$

while, for fermions, no first order correction is obtained. These results coincide with the first order terms of the well-known results of Lee and Yang; see formulas (1) and (4) for $J=0$ of ref. [16]. Nevertheless, in section 4, we discuss the validity of this first order approximation in the calculation of the matrix elements of $U_{2}$ and conclude that, for bosons, (20) is a good approximation while it is not necessarily the case for fermions.

At this point, it is convenient to introduce the free spherical waves $\mid j_{k, l, m}^{(0)}>$ associated with the relative motion of the particles, with wave functions [:

$$
<\mathbf{r} \mid j_{k, l, m}^{(0)}>=j_{l}(k r) Y_{l}^{m}(\widehat{r})
$$

(with standard notation; $j_{l}$ is a spherical Bessel function, $Y_{l}^{m}(\widehat{r})$ a spherical harmonics of the angular variables of $\mathbf{r}$ ); if $\mid \mathbf{k}>$ is a plane wave normalized in a volume $\mathcal{V}$ :

$$
\left|\mathbf{k}>=\frac{4 \pi}{\sqrt{\mathcal{V}}} \sum_{l, m}(i)^{l}\left[Y_{l}^{m}(\widehat{k})\right]^{*}\right| j_{k, l, m}^{(0)}>
$$

If we insert this equality into (17) and take into account the well-known relation:

$$
\sum_{m}\left|Y_{l}^{m}(\widehat{k})\right|^{2}=\frac{2 l+1}{4 \pi}
$$

we readily obtain the result!:

$$
a_{U}^{S, A}(k)=\sum_{l}(2 l+1)\left[1+\eta(-1)^{l}\right] \times a_{U}^{(l)}(k)
$$

\footnotetext{
${ }^{5}$ To normalize these functions (with a Dirac function of $k$ vectors and Kronecker delta's of $l$ and $m$ ), it would be necessary to multiply all the $j_{k, l, m}^{(0)}(\mathbf{r})$ 's by factors $k \sqrt{2 / \pi}$; this operation is not necessary here.

${ }^{6}$ We assume rotational invariance, so that the matrix elements of $\bar{U}_{2}(1,2)$ are diagonal in $l$ and $m$.
} 
with:

$$
a_{U}^{(l)}(k)=-\frac{2 \pi}{\lambda_{T}^{2}} e^{\beta \hbar^{2} k^{2} / m}<j_{k l m}^{(0)}\left|\left[U_{2}\right]_{r e l}\right| j_{k l m}^{(0)}>
$$

where $\left[U_{2}(1,2)\right]_{\text {rel }}$ has been defined in (7); rotational invariance ensures that the right hand side of (27) is independent of $m$. These results can be inserted into the integral appearing in (15) and provide an expression of the correction to the grand potential which is a direct generalization of the usual Beth Uhlenbeck formula to gases having a significant degree of degeneracy:

$$
[\log Z]_{i n t}=-\frac{\lambda_{T}^{2} \mathcal{V}}{(2 \pi)^{6}} \sum_{l}(2 l+1)\left[1+\eta(-1)^{l}\right] \times \int d^{3} k_{1} \int d^{3} k_{2} f\left(\mathbf{k}_{1}\right) f\left(\mathbf{k}_{2}\right) \times a_{U}^{(l)}(k)
$$

For comparison, we recall the explicit expression of this formula:

$$
[\log Z]_{\text {int }}^{\text {B.U. }}=\frac{2^{3 / 2}}{2 \pi} z^{2} \frac{\mathcal{V}}{\lambda_{T}} \sum_{l}(2 l+1)\left[1+\eta(-1)^{l}\right] \int d k \mathrm{e}^{-\beta \hbar^{2} k^{2} / m} k \delta_{l}(k)
$$

(we temporarily ignore possible bound states), where $\lambda_{T}$ is defined in (16). As already discussed in [6], if we replace each of the two $f$ 's in (28) by their low density limit $e^{\beta\left(\mu-\hbar^{2} k^{2} / 2 m\right)}$, the integration over $d^{3} K$ of a Gaussian function introduces a factor $8^{3 / 2}\left[\pi / \lambda_{T}\right]^{3}$ and, after some algebra, we recover (29). In other words, the following substitutions are necessary to obtain (28) from the usual Beth Uhlenbeck formula ๆ:

$$
\left\{\begin{array}{c}
\mathrm{e}^{-\beta \hbar\left[\left(\mathbf{k}_{1}\right)^{2}+\left(\mathbf{k}_{1}\right)^{2}\right] / 2 m} \Rightarrow z^{-2} f\left(\mathbf{k}_{1}\right) f\left(\mathbf{k}_{2}\right) \\
\delta_{l}(k) \Rightarrow-k a_{U}^{(l)}(k)
\end{array}\right.
$$

In equation (10), the first of these substitutions amounts to adding the terms in $\eta f$.

\subsection{Bound states}

The operator $\left[U_{2}\right]_{\text {rel }}^{S, A}$ may be written as the sum of the contributions of bound states and of the continuum:

$$
\left[U_{2}\right]_{r e l}^{S, A}=\sum_{n}\left|\Phi_{n}\right\rangle\left\langle\Phi_{n}\right| \mathrm{e}^{-\beta E_{n}}+\text { continuum }
$$

\footnotetext{
${ }^{7}$ There are several equivalent ways to write the Beth Uhlenbeck formula; for instance, an integration by parts allows one to replace the product $k \delta_{l}(k)$ by the derivative $d \delta_{l}(k) / d k$ while the coefficient $1 / \lambda_{T}$ is replaced by $\pi / \lambda_{T}^{3}$. Under these conditions, the second line of (30) becomes $d \delta_{l}(k) / d k \Rightarrow-\left(k \lambda_{T}\right)^{2} a_{u}^{(l)}(k) / \pi$. In other words, the correspondence between ou result and the Beth Uhlenbeck formula depends on the way the latter is written.

The two functions $k \delta_{l}(k)$ and $-k^{2} a_{U}^{(l)}(k)$ are not necessarily equal but, when multiplied by a Gaussian function $e^{-\beta \hbar^{2} \mathbf{k}^{2} / m}$, have the same integral over $d^{3} k$.
} 
where the $\left|\Phi_{n}\right\rangle$ 's are the kets associated with the eigenstate of the relative motion of the particles with (negative) energy $-E_{n}$ (with appropriate symmetry for the statistics of the particles). It is therefore not difficult make the contribution of bound states in (15) explicit, which provides the following term:

$$
\begin{aligned}
{[\log Z]_{\text {int }}^{\text {bound }}=(2 \pi)^{-6} \mathcal{V}^{2} \int d^{3} K \int d^{3} q f\left(\frac{\mathbf{K}}{2}+\mathbf{q}\right) f\left(\frac{\mathbf{K}}{2}-\mathbf{q}\right) \times } \\
\times \sum_{n}\left|\left\langle\mathbf{q} \mid \Phi_{n}\right\rangle\right|^{2} \mathrm{e}^{\beta\left(E_{n}+\hbar^{2} q^{2} / m\right)}
\end{aligned}
$$

where ; because these kets have a finite range, and because the plane waves $\mid \mathbf{q}>$ are normalized in a macroscopic volume $\mathcal{V}$, the product $\mathcal{V}\left|\left\langle\mathbf{q} \mid \Phi_{n}\right\rangle\right|^{2}$ is independent of $\mathcal{V}$ in the thermodynamic limit, as necessary to obtain an extensive correction to the grand potential. If we rewrite the integral of (32) in the form:

$$
\int d^{3} K \int d^{3} q e^{-\beta \hbar^{2} \mathbf{K}^{2} / 4 m}\left[1+\eta f\left(\frac{\mathbf{K}}{2}+\mathbf{q}\right)\right] \times\left[1+\eta f\left(\frac{\mathbf{K}}{2}-\mathbf{q}\right)\right] \sum_{n}\left|\left\langle\mathbf{q} \mid \Phi_{n}\right\rangle\right|^{2} \mathrm{e}^{\beta E_{n}}
$$

we may distinguish between two contributions in the correction:

$$
[\log Z]_{\text {int }}^{\text {bound }}=[\log Z]_{\text {B.U. }}^{\text {bound }}+[\log Z]_{\text {stat. }}^{\text {bound }}
$$

The first contribution is obtained by ignoring in (33) the $\eta f^{\prime}$ 's inside the brackets, which allows one to integrate over $d^{3} K$; using the closure relations over the plane waves $|\mathbf{q}\rangle$ and the normalization of the bound states $\left|\Phi_{n}\right\rangle$ then provides the following result for this first contribution of the bound states:

$$
[\log Z]_{B . U .}^{b o u n d}=2^{3 / 2}\left[\lambda_{T}\right]^{-3} \mathcal{V} \sum_{n} \mathrm{e}^{\beta E_{n}}
$$

which is identical to the term corresponding to bound states in the usual Beth Uhlenbeck formula. The second contribution arises from the rest of the product of the brackets and is equal to:

$$
\begin{aligned}
{[\log Z]_{\text {stat. }}^{\text {bound }}=} & (2 \pi)^{-6} \mathcal{V}^{2} \int d^{3} K \int d^{3} q \mathrm{e}^{-\beta \hbar^{2} \mathbf{K}^{2} / 4 m}\left[f\left(\frac{\mathbf{K}}{2}+\mathbf{q}\right) f\left(\frac{\mathbf{K}}{2}-\mathbf{q}\right)+\right. \\
& \left.+\eta f\left(\frac{\mathbf{K}}{2}+\mathbf{q}\right)+\eta f\left(\frac{\mathbf{K}}{2}-\mathbf{q}\right)\right] \sum_{n}\left|\left\langle\mathbf{q} \mid \Phi_{n}\right\rangle\right|^{2} \mathrm{e}^{\beta E_{n}}
\end{aligned}
$$

It accounts for exchange effects between bound states and continuum states which explains the appearance of the scalar product $\left\langle\mathbf{q} \mid \Phi_{n}\right\rangle$ of a free plane wave

\footnotetext{
${ }^{8}$ The exchange effects between bound states themselves will be investigated in another article with the study of pair condensation (BCS condensation for fermions); they are higher order in $U_{2}$.
} 
and a bound state wave function; the factors $e^{\beta E_{n}}$ correspond physically to the Boltzmann distribution of the populations of the bound states.

If we assume that the momenta in the continuum, which have values that are limited by the presence of the $f$ 's under the integral, are much smaller than those contained in the bound states $\left|\Phi_{n}\right\rangle$ 's, we can replace the product $\left\langle\mathbf{q} \mid \Phi_{n}\right\rangle$ by $\left\langle\mathbf{q}=\mathbf{0} \mid \Phi_{n}\right\rangle$; this shows that the effect of statistics is more important for bound states with a wave function with a significant integral over space, as for instance the ground state wave function which has no node; states with rapidly oscillating wave functions give almost no correction to the usual Beth Uhlenbeck formula. Generally speaking, for bosons, the effect of statistics is always to increase the Beth Uhlenbeck term (35). For fermions, the situation is more complicated: if the gas is only slightly degenerate one has $f^{2}<f$ so that the terms in $\eta$ dominate in (36), leading to a decrease of the effects of bound states; but if the gas is strongly degenerate, there seems to be no general rule, and exchange of bound states with the continuum may either enhance or reduce their contribution.

\subsection{Spins}

An easy generalization is to include spins; the only difference is that all the traces must now also include spin states. It is then convenient to replace the functions $f(\mathbf{k})$ of momentum by spin operators $f_{S}(\mathbf{k})$ (corresponding to two by two matrices for spin $1 / 2$ particles) which are functions of $\mathbf{k}$ and are defined as:

$$
\left\langle m_{S}\left|f_{S}(\mathbf{k})\right| m_{S}^{\prime}\right\rangle=\left\langle m_{S}, \mathbf{k}\left|\frac{z e^{-\beta H_{1}}}{1-\eta z e^{-\beta H_{1}}}\right| m_{S}^{\prime}, \mathbf{k}\right\rangle
$$

where $H_{1}$ is the one particle hamiltonian, including kinetic energy as well as coupling of the spins to the magnetic field (if the particles carry magnetic moments). Similarly, because now the exchange of particles must also include their spin states, $a_{U}^{S, A}(k)$ becomes an operator $\Sigma_{S}(k)$ which acts in the space of the states of two spins:

$$
\Sigma_{S}(k)=\sum_{l=0}^{\infty}(2 l+1) a_{U}^{(l)}(k)\left[1+\eta(-1)^{l} P_{e x}^{S}\right]
$$

where $P_{e x}^{S}$ is the exchange operators of two spins. This leads to the following generalization of (15):

$$
[\log Z]_{i n t}=-\frac{\lambda_{T}^{2} \mathcal{V}}{(2 \pi)^{6}} \int d^{3} k_{1} \int d^{3} k_{2} \operatorname{Tr}_{S_{1}, S_{2}}\left\{f_{S}\left(\mathbf{k}_{1}\right) f_{S}\left(\mathbf{k}_{2}\right) \Sigma_{S}(k)\right\}
$$

\section{Spin 1/2 particles}

We now apply the preceding calculation to the study of the magnetic susceptibility of a dilute gas of fermions or bosons with spin $1 / 2$ (spin polarized atomic 
hydrogen provides an example of spin 1/2 bosons [17, 18]). We assume that the one particle hamiltonian is:

$$
H_{1}=\frac{\mathbf{P}^{2}}{2 m}-\frac{\hbar \omega_{0}}{2} \sigma_{z}
$$

where $\omega_{0} / 2 \pi$ is the Larmor frequency in the (homogeneous) magnetic field and $\sigma_{z}$ the Pauli matrix (operator) associated with the component of the spin along the field; we set:

$$
z_{ \pm}=\mathrm{e}^{\beta\left(\mu \pm \hbar \omega_{0} / 2\right)}=z \mathrm{e}^{ \pm \beta \hbar \omega_{0} / 2}
$$

which gives the following values for the matrix elements Đ of $f_{S}(\mathbf{k})$ defined in (37):

$$
f_{ \pm}(\mathbf{k})=\frac{z_{ \pm} \mathrm{e}^{-\beta \hbar^{2} k^{2} / 2 m}}{1-\eta z_{ \pm} \mathrm{e}^{-\beta \hbar^{2} k^{2} / 2 m}}
$$

For short we will write:

$$
f_{ \pm}(1)=f_{ \pm}\left(\mathbf{k}_{1}\right) \quad f_{ \pm}(2)=f_{ \pm}\left(\mathbf{k}_{2}\right)
$$

With this notation we have:

$$
\begin{gathered}
{[\log Z]_{i g}=-\eta \frac{\mathcal{V}}{8 \pi^{3}} \int d^{3} k_{1} \log \left\{\left[1-\eta z_{+} \mathrm{e}^{-\beta \hbar^{2} k_{1}^{2} / 2 m}\right]\left[1-\eta z_{-} \mathrm{e}^{-\beta \hbar^{2} k_{1}^{2} / 2 m}\right]\right\}} \\
=\eta \frac{\mathcal{V}}{8 \pi^{3}} \int d^{3} k_{1} \log \left\{\left[1+\eta f_{+}(1)\right]\left[1+\eta f_{-}(1)\right]\right\}
\end{gathered}
$$

For a gas at equilibrium the operators $f_{S}(\mathbf{k})$ are diagonal in the basis corresponding to a quantization axis parallel to the magnetic field; we then have:

$$
\begin{aligned}
\operatorname{Tr}_{S_{1}, S_{2}}\left\{f_{S}\left(\mathbf{k}_{1}\right) f_{S}\left(\mathbf{k}_{2}\right)\right\} & =\operatorname{Tr}_{S_{1}}\left\{f_{S}\left(\mathbf{k}_{1}\right)\right\} \times \operatorname{Tr}_{S_{2}}\left\{f_{S}\left(\mathbf{k}_{2}\right)\right\} \\
& =\left[f_{+}(1)+f_{-}(1)\right]\left[f_{+}(2)+f_{-}(2)\right]
\end{aligned}
$$

as well as:

$$
\operatorname{Tr}_{S_{1}, S_{2}}\left\{f_{S}\left(\mathbf{k}_{1}\right) f_{S}\left(\mathbf{k}_{2}\right) P_{e x}^{S}\right\}=f_{+}(1) f_{+}(2)+f_{-}(1) f_{-}(2)
$$

(the latter result arises because the trace gets non zero contributions only from the two spin states $|+,+\rangle$ and $|-,-\rangle$, which are invariant under the effect of $\left.P_{e x}^{S}\right)$. We therefore have:

$$
\begin{aligned}
{[\log Z]_{i n t}=-\frac{\lambda_{T}^{2} \mathcal{V}}{(2 \pi)^{6}} \int d^{3} k_{1} \int d^{3} k_{2} } & \left\{a_{U}^{S, A}(k)\left[f_{+}(1) f_{+}(2)+f_{-}(1) f_{-}(2)\right]\right. \\
+ & \left.2 a_{U}(k) f_{+}(1) f_{-}(2)\right\}
\end{aligned}
$$

\footnotetext{
${ }^{9}$ We remind the reader that, except for an ideal gas, the $f_{ \pm}$'s are not the populations of the one body density operators, but differ from them by density corrections [25].
} 
where:

$$
k=\frac{\left|\mathbf{k}_{1}-\mathbf{k}_{2}\right|}{2}
$$

while $a_{U}^{S, A}(k)$ is defined by (17) - or equivalently (26) - while $a_{U}(k)$ is the unsymmetrized version $\square$ of the Ursell length:

$$
a_{U}(k)=-\frac{\mathcal{V}}{2 \lambda_{T}^{2}}<\mathbf{k}\left|\bar{U}_{2}\right| \mathbf{k}>=\sum_{l=0}^{\infty}(2 l+1) a_{U}^{(l)}(k)
$$

(it would correspond to distinguishable particles). If, as in the beginning of section 2.3, we treat the pseudopotential (19) to first order, for fermions we obtain $a_{U}^{A}(k)=0$ and $a_{U}(k)=a$; the first order correction is now given by:

$$
[\log Z]_{i n t} \simeq-2 \frac{a \mathcal{V}}{\lambda_{T}} \times g_{3 / 2}(-z)
$$

which coincides with the result of Lee and Yang (equation (1) of [16] for $J=1 / 2$ ); but, again, we note that a critical discussion of this first order calculation is made in section 14 which shows that, for fermions, the results of the Ursell approach may be different from those of a pseudopotential theory.

\subsection{Density of particles and of energy; magnetization.}

The number density of the gas is obtained from the relation:

$$
n=\frac{\langle N\rangle}{\mathcal{V}}=\mathcal{V}^{-1} z \frac{\partial}{\partial z}[\log Z]
$$

Similarly, then density of internal energy is given by:

$$
w=\frac{\langle U\rangle}{\mathcal{V}}=-\mathcal{V}^{-1} \frac{\partial}{\partial \beta}[\log Z]
$$

while the "magnetization" $\square$ is equal to:

$$
M=\frac{2}{\beta \hbar} \frac{\partial}{\partial \omega_{0}}[\log Z]
$$

\footnotetext{
${ }^{10}$ Note the factor $1 / 2$ which does not appear in (17); we choose this convention since the same factor $1 / 2$ appears in the definition (3) of the symmetrized version of $U_{2}$; in this way, if exchange effects are ignored (high temperature limit for instance), the various Ursell lengths become equal.

${ }^{11}$ What we call here magnetization is not a real magnetic moment (ampere square meter) but a dimensionless number equal to the sum of the average values of $\sigma_{z}$ of all atoms; in other words, the maximum value of $M$ (complete spin polarization, all spins parallel) is equal to the total number of particles.
} 
We therefore have to vary either $z$, or $\beta$, or $\omega_{0}$ in formulas (44) and (47); we can then use the simple relations:

$$
d z_{ \pm}=z_{ \pm}\left(\frac{d z}{z} \pm \frac{\hbar \omega_{0}}{2} d \beta \pm \beta \hbar \frac{d \omega_{0}}{2}\right)
$$

and:

$$
z_{ \pm} \frac{\partial f_{ \pm}}{\partial z_{ \pm}}=f_{ \pm}\left[1+\eta f_{ \pm}\right]
$$

as well as:

$$
\frac{\partial f_{ \pm}}{\partial \beta}=f_{ \pm}\left[1+\eta f_{ \pm}\right]\left[-\frac{\hbar^{2} k^{2}}{2 m} \pm \frac{\hbar \omega_{0}}{2}\right]
$$

We then obtain, by making use of the symmetry in the indices 1 and 2 :

$$
\begin{gathered}
n=(2 \pi)^{-3} \int d^{3} k_{1}\left[f_{+}(1)+f_{-}(1)\right]+ \\
-\frac{\lambda_{T}^{2}}{(2 \pi)^{6}} \int d^{3} k_{1} \int d^{3} k_{2}\left\{2 a_{U}^{S, A}(k) f_{+}(1) f_{+}(2)\left[1+\eta f_{+}(1)\right]+i d .\left(f_{+} \Leftrightarrow f_{-}\right)+\right. \\
\left.+2 a_{U}(k) f_{+}(1) f_{-}(2)\left[2+\eta f_{+}(1)+\eta f_{-}(2)\right]\right\}
\end{gathered}
$$

where $i d .\left(f_{+} \Leftrightarrow f_{-}\right)$symbolizes the same expression where $f_{+}$and $f_{-}$are interchanged. The calculation of $M$ is almost the same, except that now, because of (54), $f_{+}$and $f_{-}$introduce different signs; the result is:

$$
\begin{gathered}
\frac{M}{\mathcal{V}}=(2 \pi)^{-3} \int d^{3} k_{1}\left[f_{+}(1)-f_{-}(1)\right]+ \\
-\frac{\lambda_{T}^{2}}{(2 \pi)^{6}} \int d^{3} k_{1} \int d^{3} k_{2}\left\{2 a_{U}^{S, A}(k) f_{+}(1) f_{+}(2)\left[1+\eta f_{+}(1)\right]-i d \cdot\left(f_{+} \Leftrightarrow f_{-}\right)+\right. \\
\left.+2 \eta a_{U}(k) f_{+}(1) f_{-}(2)\left[f_{+}(1)-f_{-}(2)\right]\right\}
\end{gathered}
$$

Finally, the calculation of the internal energy $w$ provides the result:

$$
w=w_{E}+w_{M}
$$

where $w_{E}$ is the density of energy associated with the external variables of the particles (kinetic and potential energy):

$$
\begin{aligned}
& w_{E}=(2 \pi)^{-3} \int d^{3} k_{1}\left[f_{+}(1)+f_{-}(1)\right] \frac{\hbar^{2} k_{1}^{2}}{2 m}+ \\
& -\frac{\lambda_{T}^{2}}{(2 \pi)^{6}} \int d^{3} k_{1} \int d^{3} k_{2}\left\{2 a_{U}^{S, A}(k) f_{+}(1) f_{+}(2)\left[1+\eta f_{+}(1)\right] \frac{\hbar^{2} k_{1}^{2}}{2 m}+i d \cdot\left(f_{+} \Leftrightarrow f_{-}\right)+\right. \\
& \left.+2 a_{U}(k) f_{+}(1) f_{-}(2)\left[\left[1+\eta f_{+}(1)\right] \frac{\hbar^{2} k_{1}^{2}}{2 m}+\left[1+\eta f_{-}(2)\right] \frac{\hbar^{2} k_{2}^{2}}{2 m}\right]\right\}
\end{aligned}
$$


while $w_{M}$ is the density of magnetic energy:

$$
w_{M}=-\frac{\hbar \omega_{0}}{2} \frac{M}{\mathcal{V}}
$$

We now assume that the magnetic field is sufficiently low so that the preceding expressions may be limited to their first order expansion in $\omega_{0} \simeq 0$. We then use the relations:

$$
f_{ \pm} \simeq f+\frac{\partial f_{ \pm}}{\partial z_{ \pm}} \frac{\partial z_{ \pm}}{\partial \omega_{0}} \omega_{0}=f \pm z \frac{\partial f}{\partial z} \frac{\beta \hbar \omega_{0}}{2}=f\left[1 \pm \beta \hbar \frac{\omega_{0}}{2}[1+\eta f]\right]
$$

which provide:

$$
\begin{gathered}
n\left(\omega_{0}\right) \simeq n(0)=\left(4 \pi^{3}\right)^{-1} \int d^{3} k_{1} f(1)-\frac{\lambda_{T}^{2}}{(2 \pi)^{6}} \int d^{3} k_{1} \int d^{3} k_{2} f(1) f(2) \times \\
\times 4[1+\eta f(1)]\left[a_{U}^{S, A}(k)+a_{U}(k)\right]
\end{gathered}
$$

and for the zero field magnetic susceptibility of the gas:

$$
\begin{aligned}
\chi=\frac{M}{d \omega_{0} \mathcal{V}} & =\beta \hbar \int d^{3} k_{1} f(1)[1+\eta f(1)]-\frac{\lambda_{T}^{2}}{(2 \pi)^{6}} \beta \hbar \int d^{3} k_{1} \int d^{3} k_{2} f(1) f(2) \times \\
\times & \left\{2 a_{U}^{S, A}(k)[1+\eta f(1)][2+2 \eta f(1)+\eta f(2)]+\right. \\
& \left.+a_{U}(k)\left[[f(1)-f(2)]^{2}+2 \eta f(1)[1+\eta f(1)]\right]\right\}
\end{aligned}
$$

\subsection{Physical discussion}

Equations (57) and (58) show how quantum statistical effects modify the equation of state and the magnetic susceptibility of a gas. What determines the properties of the dilute system is not directly the matrix elements of the potential $V_{\text {int }}$, but those of the operator $\bar{U}_{2}$. Of course, if the potential is weak, the calculation may be limited to first order perturbation theory, so that $\bar{U}_{2}$ may be replaced by $-\beta V_{\text {int }}$; the two operators then become equivalent. But, in more realistic situations, for instance in atomic systems where the interaction potential at short relative distances becomes very large, the effects of the potential cannot be treated to first order. An obvious difference between $\bar{U}_{2}$ and $V_{\text {int }}$ is that the former depends on one more characteristic length than the potential, namely the thermal wavelength $\lambda_{T}$, so that one can expect that its range will depend explicitly on the temperature. It is sometimes argued that a description of the interactions in terms of the scattering length is sufficient at very low temperatures, because the range of the potential $V_{\text {int }}$ remains always much smaller than the de Broglie wavelengths of the particles. As far as the matrix elements of $\bar{U}_{2}$ are concerned, 
this is of course true if the interaction potential is treated to first order only but, precisely at very low temperatures, the higher order terms become significant and $\bar{U}_{2}$ may acquire a range that exceeds by far that of the potential itself. We will see examples of this phenomenon in section 1 .

We now discuss the limit of validity of the results obtained in the preceding section or, since they are equivalent, of equations (14) and (39). If the particles are bosons, our theory is limited to gases which are not too close to Bose Einstein condensation - while of course the degeneracy may be much more pronounced than for the Beth Uhlenbeck formula since $z$ does not have to be small. The reason is that, when a system of bosons approaches the region of quantum condensation, the distribution function $f$ starts to build up a singularity at low energies. One can then see that, when $z \rightarrow 1$, the partition function becomes more and more sensitive to terms of higher and higher order in $\bar{U}_{2}$, in $\bar{U}_{3}, \bar{U}_{4}$, etc. , since they contain larger and larger number of functions $f$ (or of factors $1+\eta f$ ). In other words, terms which normally remain small corrections become dominant when the point of Bose Einstein condensation is approached; see the discussion already given in [7], where it is emphasized[ए] that a theory limited to first order in $\bar{U}_{2}$ would predict the disappearance of the Bose Einstein condensation phenomenon and its replacement by a simple crossover between two regimes.

For fermions, the discussion is different since no special phenomenon takes place when $z$ reaches one; when the gas is cooled at constant density, this merely corresponds to a cross over region where the gas is becoming degenerate. When the temperature is decreased even more, a stronger degeneracy builds up while $z=\mathrm{e}^{\beta \mu}$ becomes larger and larger $(\beta$ increases while $\mu$ remains almost constant if the density is fixed). It is therefore clear that $z$ itself can not be an expansion parameter in this region; but our perturbation series is not a $z$ expansion and it may still converge for degenerate systems, provided the matrix elements of $\bar{U}_{2}$ are sufficiently small. In the next section, we will see that these matrix elements are equal to the product $\lambda_{T}^{2} a_{U}$, where $\lambda_{T}$ is the thermal wave length and $a_{U}$ is some microscopic length (the Ursell length) that we will define more precisely later. Let us for instance discuss (57) where the interaction corrections involve a double integral over two momenta. The first introduces the number density $n$; the second contains a function $f(1-f)$, which in the limit of low temperatures is non zero only in an energy slice of width $\beta^{-1}$ at the surface of the Fermi sphere; the corresponding width $\Delta k$ in terms of momentum is given by:

$$
\frac{\hbar^{2} k_{F} \Delta k}{m}=\beta^{-1}
$$

(where $\hbar k_{F}$ is the momentum at the Fermi surface) so that the result of these

\footnotetext{
${ }^{12}$ Unfortunately, in [7] an assumption of this discussion is not made explicit, namely the fact that the matrix elements of $U_{2}$ should be positive (dominant character of the attractive interactions in the matrix element).
} 
integrations is:

$$
n\left(\lambda_{T}^{2} a_{U}\right) \times 4 \pi k_{F}^{2} \frac{2 \pi}{k_{F} \lambda_{T}^{2}}=2 n k_{F} a_{U}
$$

The small parameter of the expansion $\left[3\right.$ is therefore the product $k_{F} a_{U}$, which is not very different from $n^{1 / 3} a_{U}$ for degenerate gases, and indeed remains small if the average distance between the particles is much larger than $a_{U}$. This result is not surprising: indeed, in the usual theory of dilute non ideal Fermi gases [10], the expansion parameter is independent of the temperature, which plays no particular role as long as the system is strongly degenerate.

One might be tempted at this point to conclude that the generalized Beth Uhlenbeck formula is valid for arbitrary degeneracy of a fermionic system; nevertheless, in the next section, we will see that the factor $a_{U}$ itself may become very large at very low temperatures, which in turn increases the expansion parameter and, automatically, limits the validity of the expansion at some point. We will assume that this somewhat unexpected phenomenon is a precursor of BCS pair condensation; if this is the case, the low temperature limit of the validity of the generalized Beth Uhlenbeck is that the gas should remain a normal Fermi gas and, even, not to be too close to condensation. In general, for bosons as well as for fermions, we can therefore conclude that our calculations remain valid as long as Bose Einstein or BCS condensation is not too close.

\section{Matrix elements of $\bar{U}_{2}$}

We now study in more detail the values of the basic ingredient that we use to describe the effects of the interactions on the thermodynamic properties of a quantum gas, namely the diagonal matrix element of $\bar{U}_{2}^{S, A}$ - together with those of the un-symmetrized operator if the particles have spins, see section 3. A natural question then is the following: to determine these coefficients, is it sufficient to characterize the potential in terms of its binary collision phase shifts, which determine the asymptotic behavior of interacting wave functions, or is it also necessary to include some information on the behavior of the wave functions at short relative distances, inside the potential? For bosons at very low temperatures, is it possible, even more simply, to reason in terms of the scattering length only?

We have already mentioned in the introduction that, in the literature on low temperature dilute systems, it is often considered as physically obvious that the short range properties of the potential play no role in the thermodynamics; it is

\footnotetext{
${ }^{13}$ We note in passing that the phase occupation factor $(1-f)$ plays an essential role in this argument. This factor occurs in all expressions, for instance, in (58), the bracket $\left[f_{+}(1)-f_{-}(2)\right]$ may be written as $\left[f_{+}(1)-1+1-f_{-}(2)\right]$. If we had ignored this factor, we would have found $n a_{U} \lambda_{T}^{2}$ as the expansion parameter, which is a temperature dependent factor (as in the usual Beth Uhlenbeck formula).
} 
then possible to replace the real potential by an effective potential (or pseudopotential) which has the expression already given in (19) in terms of the scattering length $a$. With a potential having zero range, it becomes of course meaningless to take into account the distortions of the wave functions inside the potential, in other words the contribution of particles "in the middle of a collision". But with real potentials, it is not obvious that these contributions are non existent, in particular since statistical effects arising from particle exchange are expected to be more important when the particles are close. We discuss here this question in the context of the generalized Beth Uhlenbeck formula that we have obtained, with the help of simple examples such as a step like potential mimicking a real interaction potential, or even hard spheres. We first discuss the Ursell length $a_{U}(k)$, which we have already introduced above, and which depends on the temperature while $a$ does not.

\subsection{Ursell length}

If the interaction potential $V_{\text {int }}$ were sufficiently weak, we could use a first order perturbation expression of the Ursell operator:

$$
U_{2}=-\int_{0}^{\beta} d \beta^{\prime} \mathrm{e}^{-\beta^{\prime} H_{0}} V_{i n t} e^{\left(\beta^{\prime}-\beta\right) H_{o}}+\ldots
$$

which would lead to the following expression:

$$
<\mathbf{k}\left|\bar{U}_{2}\right| \mathbf{k}>=-\beta<\mathbf{k}\left|V_{\text {int }}\right| \mathbf{k}>+\ldots
$$

Inserting (19) into this result provides:

$$
<\mathbf{k}\left|\bar{U}_{2}\right| \mathbf{k}>=-\beta \frac{4 \pi \hbar^{2} a}{m \mathcal{V}}=-\frac{2 a \lambda_{T}^{2}}{\mathcal{V}}
$$

where $\mathcal{V}$ is the macroscopic volume and $\lambda_{T}$ the thermal wavelength (16).

By analogy with this first order calculation, it is convenient to characterize the diagonal matrix elements in terms of a length, $a_{U}(k)$, which we call the Ursell length and which we have defined in (49) as:

$$
a_{U}(k)=-\frac{\mathcal{V}}{2 \lambda_{T}^{2}}<\mathbf{k}\left|\bar{U}_{2}\right| \mathbf{k}>
$$

This very definition ensures that, within the theory of the pseudopotential, $a_{U}(k)$ is exactly equal to the scattering length $a$. This length therefore provides a convenient tool for a discussion of the validity of this approximation: as long as $a_{U}(k)$ remains very close to $a$ for all relevant values of $\mathbf{k}$, the theory of pseudopotentials and ours provide strictly equivalent results. For identical particles, what is needed is the symmetrized Ursell length, already defined in (17) and (26), which 
allows us to write the correction to the grand potential for spinless particles in the form (15). This equation (15) expresses that the symmetrized Ursell length gives, within the numerical factor in front of this integral, the crossed contribution of two velocity classes $\mathbf{k}_{1}$ and $\mathbf{k}_{2}$ to the grand potential of the system (its pressure). If the particles have spins, we have to use (39) and (38).

In section 2.3, we introduced spherical orbital variables from the free spherical waves $\mid j_{k, l, m}^{(0)}>$. At this stage, it is convenient to use spherical waves that are normalized in a sphere of volume $\mathcal{V}=4 \pi R^{3} / 3$. We therefore introduce a new notation, $\mid \varphi_{k l m}^{(0)}>$, but these kets are simply proportional to the $\mid j_{k l m}^{(0)}>$ :

$$
\left|\varphi_{k l m}^{(0)}>=x_{k l}\right| j_{k l m}^{(0)}>
$$

where $x_{k l}$ is a normalization coefficient which in the limit of large values of the product $k R$ is equal to:

$$
x_{k l}=\sqrt{\frac{2 k^{2}}{R}}
$$

Introducing in the same way the normalized kets $\mid \varphi_{k^{\prime} l m}>$ in the presence of the interaction potential, we can write:

$$
a_{U}^{(l)}(k)=-\frac{2 \pi}{\lambda_{T}^{2}}\left[x_{k l}\right]^{-2}\left[\sum_{k^{\prime}}\left|<\varphi_{k, l, m}^{(0)}\right| \varphi_{k^{\prime}, l, m}>\left.\right|^{2} e^{-\beta\left[e\left(k^{\prime}\right)-e(k)\right]}-1\right]
$$

where $e(k)$ and $e\left(k^{\prime}\right)$ are the energies of the free and interacting states.

In what follows we discuss the values of the Ursell length and its $\mathbf{k}$ dependence with the help of a few examples.

\subsection{Steplike potential}

In order to simplify our discussion, we now consider an interaction potential made of a hard core of diameter $x b$ (with $x \leq 1$ ) and of an attractive part $\left(V_{\text {int }}=-V_{0}\right)$ from relative distance $r=x b$ to $r=b$; see figure 1. Our discussion is in fact more general, but this kind of simplified potential is a convenient way to mimic the effects of a more realistic interaction potential, containing strong repulsion at short distances and Van der Waals attraction at large distances. For instance, it is not difficult to find a relation between $V_{0}, x$ and $b$ which ensures that the scattering length of this potential vanishes (compensation of the effects of attraction and repulsion at low energies). Does this imply that the matrix elements of $U_{2}$, that is the Ursell length $a_{U}(k)$, also vanish? Not in general, since

the scalar products $<\varphi_{k, l, m}^{(0)} \mid \varphi_{k^{\prime}, l, m}>$ in (73) are not only sensitive to the changes of the interacting wave functions outside of the potential (which do not occur if the scattering length vanishes) but also to their values inside the potential. In other words, for a degenerate dilute gas, all the effects of the potential are not necessarily contained in the scattering phase shifts. 
We nevertheless note that, when the range $b$ of the potential tends to zero, the corrections to the scalar products are necessarily of third order in $b$ (at least), while first order terms occur in the phase shifts (for instance, at very low temperatures, the Beth Uhlenbeck formula predicts a correction which is proportional to $b \lambda_{T}^{2}$ ). Therefore, if the potential range is sufficiently small (compared to the two other microscopic distances, the average distance between particles $n^{-1 / 3}$ and $\lambda_{T}$ ), one may limit the calculation to first order in $b$ so that the contribution of the distortion of the wave functions inside the potential may be ignored. Within this approximation, all effects of the potential on the thermodynamic properties are indeed contained in the collision phase shifts. A brief similar discussion in terms of "shape dependent terms" (as opposed to phase shift dependent terms) appears in the two last paragraphs of section 5 of ref. 19

Consequently, in all calculations of the thermodynamic quantities which are limited to first order in the potential range, it is sufficient to characterize the potential by its long distance effects on the wave function (phase shifts) only, while short range effects are irrelevant; if all collisions take place at very low energies, all effects are then contained in the scattering length only. On the other hand, this is not necessarily the case if higher order terms in the potential range are included, and corrections which originate "inside the potential", corresponding to the contribution of particles "in the middle of a collision", may appear; see also [19]. Actually, the question remains open as to whether is would be possible, by some mathematical transformation, to express these "in potential effects" in terms of the phase shifts only; this is for instance possible in the absence of the phase occupation factors, since one then gets the usual Beth Uhlenbeck formula for which such a transformation is known. In the presence of these factors, we have made efforts to investigate the possibility that a similar simplification takes place, but we have not been able to prove it. A possible conjecture is that short range and long range effects are in general independent from each other, but this remains to be proved by a precise example.

\subsection{Hard spheres}

For hard spheres, the interacting wave function does not penetrate into the potential; this is a special case where all physical effects of the potential are necessarily contained in the collision phase shifts. The potential range $b$ coincides in this case with the scattering length $a$. But this is not sufficient to ensure that the theory of pseudopotentials should be equivalent to our results, and we now discuss this question.

\subsubsection{Analytical calculation}

In order to obtain the correction to the thermodynamic potential for hard spheres, we now perform a calculation of the scalar products which appear in (73). 
s waves For free waves, the quantification for the wave numbers is given by:

$$
k=\frac{n \pi}{R}
$$

while, for hard spheres of diameter $a$, the interacting waves satisfy the relation:

$$
k^{\prime}=\frac{n^{\prime} \pi}{R-a}
$$

where $n$ and $n^{\prime}$ are integer numbers. The scalar products of (73) then become functions of these numbers:

$$
<\varphi_{k, 0,0}^{(0)} \mid \varphi_{k^{\prime}, 0,0}>=\frac{2}{\sqrt{R(R-a)}} \int_{a}^{R} d r \sin \left(\frac{n \pi r}{R}\right) \sin \left(\frac{n^{\prime} \pi(r-a)}{R-a}\right)
$$

which, after a simple calculation, leads to:

$$
\left|<\varphi_{k, 0,0}^{(0)}\right| \varphi_{k^{\prime}, 0,0}>\left.\right|^{2}=\pi^{-2} \sin ^{2} \frac{n \pi a}{R} \frac{4 n^{\prime 2}\left[1-\frac{a}{R}\right]}{\left[n^{\prime}-n(1-a / R)\right]^{2}\left[n^{\prime}+n(1-a / R)\right]^{2}}
$$

This result, when inserted into (73) for $l=0$, provides the contributions of $\mathrm{s}$ waves to the Ursell length. Equivalent results for infinite volume (only s wave) are given by Lee and Yang [20]; we have checked that our numerical results are compatible with those of these authors. Since $n a / R=k a / \pi$, equation (77) shows that the scalar product is peaked around a value of $n^{\prime}$ given by:

$$
n^{\prime}=n-E\left(\frac{k a}{\pi}\right)
$$

where $E$ is the integer value; for low energies, $k a \ll 1$ and the preceding equation reduces to $n^{\prime}=n$.

Assume for instance that we are interested in calculating the Ursell length to first order in $a$, and in the s wave channel only. Equation (77) shows that only the term $n^{\prime}=n$ contributes and that the corresponding value is:

$$
\sin ^{2} \frac{n \pi a}{R} \frac{4 n^{2}\left[1-\frac{a}{R}\right]}{\left[\frac{n \pi a}{R}\right]^{2} n^{2}\left[2-\frac{a}{R}\right]^{2}} \simeq 1
$$

so that $(73)$ becomes to this order:

$$
a_{U}^{(0)}(k)=-\frac{2 \pi}{\lambda_{T}^{2}} \frac{R}{2 k^{2}}\left[-\beta \frac{n^{2} \hbar^{2} \pi^{2}}{m}\left(\frac{1}{(R-a)^{2}}-\frac{1}{R^{2}}\right)\right]=a
$$

The first order value is therefore merely equal to $a$; but we will see that $a_{U}^{(0)}(k)$ may strongly differ from this value if higher orders effects in the potential range are included. 
larger $l$ values Formula $(76)$ can be generalized to non-zero angular momentum $l$. The sine functions of the free and of the hard-sphere wave-functions have to be replaced by a spherical Bessel function and a linear combination of this function and a Neumann function of order $l$ respectively. After normalization we obtain for $|\mathbf{r}|>a$ :

$$
\varphi_{k^{\prime}, l, 0}(\mathbf{r})=\sqrt{\frac{2 a^{2} k^{4}}{R\left[(k a)^{2} m_{l}^{2}(k a)-a / R\right]}}\left[j_{l}\left(k^{\prime} a\right) n_{l}\left(k^{\prime} r\right)-n_{l}\left(k^{\prime} a\right) j_{l}\left(k^{\prime} r\right)\right] Y_{l}^{0}(\hat{r})
$$

$m_{l}\left(k^{\prime} a\right)$ stands for "modulus" [21, 9.2.17]:

$$
m_{l}\left(k^{\prime} a\right)=\sqrt{j_{l}^{2}\left(k^{\prime} a\right)+n_{l}^{2}\left(k^{\prime} a\right)}
$$

The quantification conditions have to be modified; (75) now becomes implicit:

$$
k^{\prime} R+\eta_{l}\left(k^{\prime}\right)=n \pi+\frac{l \pi}{2}
$$

where $\eta_{l}\left(k^{\prime}\right)$ stands for the $l$-wave phase shift for wave number $k^{\prime}$, which is given by:

$$
\eta_{l}\left(k^{\prime}\right)=\arctan \frac{j_{l}\left(k^{\prime} a\right)}{n_{l}\left(k^{\prime} a\right)}-m_{k^{\prime}} \pi
$$

In this definition we limit the values of the inverse tangent to the interval $\left[-\frac{\pi}{2} ; \frac{\pi}{2}[\right.$ and $m_{k^{\prime}}$ counts the number of times that — by increasing $k^{\prime} a$ from zero to the final value - the value of inverse tangent jumps from $\frac{\pi}{2}$ to $-\frac{\pi}{2}$. In this way we obtain $\eta_{l}$ as a continuous function of $k^{\prime}$. For large values of $k^{\prime} a$, the effects of the centrifugal barrier become negligible and the quantification condition becomes independent of $l$ so that it reduces to the $s$-wave expression (75).

The normalized free wave function is given by (23), so that we arrive at the following expression for the square of the scalar product:

$$
\left|\left\langle\varphi_{k l 0}^{(0)} \mid \varphi_{k^{\prime} l 0}\right\rangle\right|^{2}=\left[\frac{2 a(k a)\left(k^{\prime} a\right)}{R\left[\left(k^{\prime} a\right)^{2}-(k a)^{2}\right]}\right]^{2} \frac{1}{\left(k^{\prime} a\right)^{2} m_{l}^{2}\left(k^{\prime} a\right)-a / R} j_{l}^{2}(k a)
$$

\subsubsection{Numerical results}

Inserting (77) and (85) into (73) and then into (49) - or (26) - provides the Ursell length, which in turn determines the diagonal matrix element of $\bar{U}_{2}$ - or $\bar{U}_{2}^{S, A}$ for identical particles. For brevity, we just give the results of our numerical calculations of $a_{U}(k)$; in other words we only discuss interactions between particles in different spin states if the particles are indistinguishable. But there is no difficulty in treating the general case, since the calculation of $a_{U}^{S, A}(k)$ is very similar; moreover, as soon as the wave number $k$ is sufficiently small, one simply has 
$a_{U}^{S}(k) \simeq 2 a_{U}(k)$ and $a_{U}^{A}(k) \simeq 0$. The results concerning $a_{U}(k)$ are shown in figures 2 and 3 (more details about the calculations can be found in [22]). There are two parameters in the problem, $a$ and $\lambda_{T}$. As convenient dimensionless variables we choose, either the product $k \lambda_{T}$, or $k a$; the ratio $a / \lambda_{T}$ is then kept as a fixed parameter, which has small values either when the potential range is very small or when the temperature is low. Figure 2 shows that, when $a \ll \lambda_{T}$, and as long as $k \lambda_{T}$ remains smaller than 1, the Ursell length is indeed equal to $a$ (with a good accuracy), so that the theory of the pseudopotential is fully justified. For higher temperatures, we note that the low energy values of $a_{U}(k)$ become noticeably different from $a$. This is not surprising since, when $T$ increases, we progressively reach a classical regime of small wave packets; they no longer have a much larger spatial extent than the potential, while this is necessary for the approximation of the pseudopotential to apply. We therefore concentrate on low values of the ratio $a / \lambda_{T}$.

If the system is made of bosons, since the distributions $f$ 's contained in (15) have at most the same width as Gaussian thermal exponentials, the values of $k \lambda_{T}$ that are relevant in the integral are comparable to 1, or smaller; figure 2 then shows that the use of our theory or of the theory of pseudopotentials leads to the same results; see the discussion of section 2.3, in particular formula (21). But assume now that the system is made of fermions 4 , and that the temperature is progressively lowered at constant density. If the system is degenerate, the width of the functions $f$ 's is determined by the Fermi momentum $k_{F}$, which in turn depends on the density; in other words the width is practically independent of the temperature. Meanwhile, if the temperature is lowered more and more, $\lambda_{T}$ increases so that the product $k \lambda_{T}$ can take arbitrarily large values inside the integral. On the other hand, $k a$ remains limited to values smaller than 1 since, for degenerate gases where the distance between the particles is comparable to the inverse Fermi momentum in a degenerate gas, the diluteness condition $n^{1 / 3} a \ll 1$ yields:

$$
1 \gg k_{F} a \geq k a
$$

The departure from 1 of the curves of figure 2, which fall well below this value when $k \lambda_{T}$ increases, shows that significant discrepancies from a pseudopotential theory may indeed be obtained. This is even more visible in figure 3 , which uses a different variable, the product $k a$ : even for small values of the ratio $a / \lambda_{T}$, significant departures of the Ursell length from $a$ are obtained for small values of $k a$; in other words, (50) is no longer a good approximation of the generalized Beth Uhlenbeck formula. Actually, the smaller $a / \lambda_{T}$, the more pronounced these departures are; we have an illustration of the consequences of the temperature dependence of $\bar{U}_{2}$, where the effects of the potential are more and more delocalized by thermal effects while $\lambda_{T}$ increases; consequently, variations of the matrix element occur even if the range of the potential is very small and even if $k a \ll 1$.

\footnotetext{
${ }^{14}$ We are dealing here with the description of interactions of fermions in opposite spin states.
} 
In figure 4, we plot the variations of the diagonal element $<\mathbf{r}\left|\left[U_{2}\right]_{\text {rel }}\right| \mathbf{r}>$ as a function of the relative position $\mathbf{r}$; the results show clearly how the second Ursell operator acquires a longer and longer range at decreasing temperatures.

A striking feature of figure 3 is the change of sign of the Ursell length which takes place when $k a$ increases from zero. The origin of this change is understandable from (73), from which one can convince oneself that the contribution of low values of $k^{\prime}$ becomes dominant as soon as $k$ is sufficiently large; this is because, while the scalar product $\left|<\varphi_{k, l, m}^{(0)}\right| \varphi_{k^{\prime}, l, m}>\mid$ with $k^{\prime} \simeq 0$ decreases relatively slowly when $k$ increases (as $k^{-2}$ ), the exponential $e^{\beta e(k)-\beta e\left(k^{\prime}\right)}$ varies much more rapidly and so that it makes small values of $k^{\prime}$ dominate the sum. In other words, what determines the diagonal matrix element of $U_{2}$ is the contribution of interacting states that have a very small relative energy; because these states evolve more slowly in time than the free wave packets of energy $\hbar^{2} k^{2} / m$, the net differential result is equivalent to an attraction. We therefore come to the conclusion that even hard cores can result in an effective attraction between fermions of opposite spins at the surface of a Fermi sphere, provided that the temperature is sufficiently low. But other interesting features also appear; for instance there is a strong dependence of the matrix element on the relative momentum and, for some value of $k$, the Ursell length (the effective interaction) vanishes; probably more important is the fact that the effective interaction increases almost exponentially as a function of $k^{2}$, which is nothing but the square of the relative momentum of the interacting particles. This shows that the correction to the partition function is dominated by processes that take place preferably between particles having almost opposite momenta on the surface of the Fermi sphere (assuming that they have opposite spins).

\subsubsection{Validity of the Ursell expansion}

We now come back to the discussion made at the end of section 3.2 concerning the validity of the Ursell expansion for a dilute gas of fermions. The result of this discussion was that the expansion parameter is the product $n^{1 / 3} a_{U}$. This parameter would remain a constant as a function of temperature for a gas of constant density if the Ursell length did not vary too much as a function of temperature, so that the situation would be simple. But in fact, we have actually found for Fermi gases that the maximum value of $a_{U}$ becomes larger and larger at low temperatures. This automatically limits the range of validity of the first order $U_{2}$ theory to temperatures at which the relevant values of the Ursell length are not too large.

To determine a limit temperature, we will look for an asymptotic expression of the Ursell length valid for wave vectors of the magnitude of the Fermi wave vector $k \approx k_{F}$. In this case, the terms corresponding to low values of $k^{\prime}$ in the sum over the states of interacting pairs of (73) are dominant. When $k^{\prime} \ll k_{F}$ the 
square of the scalar product (85) reduces to:

$$
\left|\left\langle\varphi_{k_{F} 00}^{(0)} \mid \varphi_{k^{\prime} 00}\right\rangle\right|^{2} \approx \frac{4}{R^{2}}\left(\frac{k^{\prime}}{k_{F}^{2}}\right)^{2} \sin ^{2} k_{F} a
$$

We replace the square of the scalar product in $(73)$ by this expression and transform the sum into an integral:

$$
\begin{aligned}
a_{U}\left(k_{F}\right) & \propto-\frac{R^{2}}{k_{F}^{2} \lambda_{T}^{2}} \frac{1}{k_{F}^{4} R^{2}} \sin ^{2} k_{F} a \mathrm{e}^{\beta e_{k_{F}}} \int \mathrm{d} k^{\prime} k^{2} \mathrm{e}^{-\beta e_{k^{\prime}}} \\
& \propto-\frac{\lambda_{T}}{\left(k_{F} \lambda_{T}\right)^{6}} \sin ^{2} k_{F} a \mathrm{e}^{\beta e_{k_{F}}}
\end{aligned}
$$

where $e_{F}$ is the Fermi energy (proportional to $n^{2 / 3}$ for a strongly degenerate gas). We are interested in the limit $n^{1 / 3} a \ll 1$ or $k_{F}^{1 / 3} a \ll 1$; we can thus replace the sine by its first order approximation, which yields:

$$
\left|a_{U}\left(k_{F}\right)\right| \propto a \frac{1}{\left(k_{F} \lambda_{T}\right)^{4}} \frac{a}{\lambda_{T}} \mathrm{e}^{e_{F} / k_{B} T}
$$

The validity criterion of the Ursell expansion then becomes:

$$
n^{1 / 3} a \frac{1}{\left(k_{F} \lambda_{T}\right)^{4}} \frac{a}{\lambda_{T}} \mathrm{e}^{e_{F} / k_{B} T} \ll 1
$$

which shows that the theory is valid only when the temperature satisfies the approximate condition:

$$
k_{B} T_{C} \gtrsim \frac{e_{F}}{\log \left[n \lambda_{T}^{3}\left(\lambda_{T} / a\right)^{2}\right]}
$$

Inside the logarithmic function in the denominator, both the factor $n \lambda_{T}^{3}$ and $\left(\lambda_{T} / a\right)^{2}$ are larger than one, especially the latter for a very dilute gas; but since the logarithm has slow variations, whatever parameters we choose, in practice the limit temperature fixed by this condition is not lower than is about a tenth of Fermi temperature, a rather a high temperature compared to the transition temperature for Cooper pairing.

Our conclusion is that, for any type of interaction, and even if the density of the gas is fixed at a very low value, at sufficiently low temperature the Ursell length becomes larger and larger so that the Ursell expansion is no longer a good expansion. The maximum value of the Ursell length is obtained for pairs of fermions with opposite spins and maximum relative momenta, which corresponds to two fermions having opposite momenta on the surface of the Fermi sphere; this is reminiscent of the BCS pairing phenomenon, while the phenomenon takes place at much higher temperatures (the right hand side of (92) does not coincide with 
the standard expression of the critical temperature) so that stricto sensu it can not be called a precursor of this transition. Whether of not it is related to this transition, the change of sign of the Ursell length will have a strong effect on the two body correlation function in the system (we have seen in 25] that the second Ursell operator is closely related to the short range properties of the two body density operator). These results are also reminiscent of the well known work of Luttinger and Kohn [23] who predict the occurrence of superconductivity in purely repulsive systems, as well of the more recent work of Kagan and Chubukov [24] who also predict in this case $p$ wave superfluidity in a dilute Fermi gas. We are planning to investigate this connection in more detail in a future article.

To summarize, for both fermionic of bosonic systems, the validity of the Ursell expansion is limited, even for a very dilute gas, to temperatures sufficiently above any transition point. Mathematically, in the case of bosons the divergence of the series arises from the distribution function (or operator) $f$, while in the case of fermions its origin is the increasing value of the matrix elements of $U_{2}$ themselves.

\section{Conclusion}

Our formalism provides a systematic treatment of the interactions in a dilute gas where the basic objects are not the matrix elements of the potential itself but those of temperature dependent operators. In [25], we have shown how microscopic, short range correlations between particles could explicitly be taken into account and calculated. In the present article, we investigate the macroscopic properties of the gas by basing the discussion on expression (14), which resembles a first order perturbative expression of an energy correction, while it actually is rather different. This is mostly because the matrix element which appears in the expression is not the matrix element of the potential itself, or of some variety of pseudopotential, but the matrix element of the second Ursell operator $U_{2}$, which corresponds physically to a local Boltzmannian equilibrium. Except of course in trivial cases where the interaction potential is indeed weak for all values of the relative distances of the particles, which allows for a first order treatment of the potential, this introduces significant differences; the major reason is the temperature dependence of the matrix elements of $U_{2}$, which is in general more complex than being merely proportional to $\beta$ (as would be the case in usual perturbation theory). This matrix element is conveniently described in terms of the Ursell length. We have seen in particular that, for fermions at very low temperatures, effective attractions at the surface of a Fermi sphere may take place, independently of the repulsive or attractive character of the potential itself. Moreover, our formalism contains naturally effects such as the statistical exchange between bound molecules and free particles, which may play some role in experiments with alkali atoms at very low temperatures [12, 13, 14. 


\section{Acknowledgments}

Many useful and friendly discussions with Roger Balian took place on the subject of this article. A.M. and W.M. would like to thank the Kastler Brossel group for hospitality during stays in Paris. We are grateful for the following financial supports: NSF grant DMR-9412769 (A.M.) and INT-9015836 in conjunction with a CNRS grant (F.L. and A.M.), and NATO OTAN CRG 930644 (F.L. and W.M.).

\section{Note added}

After completion of this manuscript, we received a preprint from H. Stein et al. 26] who, in the case of bosons, find that the many body scattering length undergoes a divergence when the superfluid transition is approached, an effect similar to what we obtain for fermions. The significance of this similarity has not been examined in detail yet.

\section{Figure captions}

Figure 1: Steplike potential used to discuss compensation effects between the attractive and the repulsive part on the scattering length; $V_{0}$ is the depth of the attractive potential, $b$ its range; a hard core of range $x b$ is assumed $(x<1)$. While a relation between these three parameters can be found to make the scattering length $a$ vanish, the effect of the potential on the wave function inside its range remains significant.

Figure 2: Variations of the ratio between the Ursell length $a_{U}(k)$ and the range $a$ of the hard core potential as a function of the dimensionless variable $k \lambda_{T}$. The ratio $a / \lambda_{T}$, with values shown in the upper right, is a parameter which takes small values if either the temperature is sufficiently low or the radius of the hard cores sufficiently small.

Figure 3: Variations of the same quantity as in Figure 2, but as a function of the dimensionless variable $k a$; this representation is convenient for the discussion of fermions (where it characterizes interactions between particles in opposite spin states).

Figure 4: Variations of the diagonal matrix element $<\mathbf{r}\left|\left[U_{2}\right]_{\text {rel }}\right| \mathbf{r}>$ as a function of $\mathbf{r}$, for various values of the parameter $a / \lambda_{T}$; at low temperatures, the thermal increase of the range of the operator is clearly visible. 


\section{References}

[1] B. Kahn and G. E. Uhlenbeck. Physica, V, 399, 1938.

[2] H. D. Ursell. Proc. Cambridge Phil. Soc., 23, 685, 1927.

[3] E. Beth and G. E. Uhlenbeck. Physica, IV, 915, 1937.

[4] K. Huang. Statistical Mechanics, Wiley, 1963, § 14.3; second edition, 1987, $\S 10.3$.

[5] R. K. Pathria. Statistical Mechanics, § 9.7. Pergamon Press, Oxford, 1972.

[6] P. Grüter and F. Laloë. J. de I,Physique 5, 181, 1995.

[7] F. Laloë. In A. Griffin, D. Snoke, and S. Stringari, editors, Bose Einstein Condensation. Cambridge University Press, 1993.

[8] K. Huang. Statistical Mechanics, Wiley, 1963, § 13.3; second edition, 1987, $\S 10.5$.

[9] K. Huang and C. N. Yang. Phys. Rev., 105, 767, 1957.

[10] Landau and Lifshitz, E.M. Lifhitz and L.P. Pitaevskii, Statistical Physics, Course in theoretical physics vol. 9, §6, Pergamon Press (1980).

[11] A.A. Abrikosov, L.P. Gorkov and I.E. Dzyaloshinskii, Methods of Quantum Field Theory in Statistical Physics, Dover (N.Y) 1975, §§ 1.4, 1.5, 3.15.

[12] M. H. Anderson, J. R. Ensher, M. R. Matthews, C. E. Wieman and E.A. Cornell, Science, page 198, 1995.

[13] C. C. Bradley, C. A. Sackett, J. J. Tollett, and R. G. Hulet. Phys. Rev. Let., 75, 1687, 1995.

[14] K. B. Davis, M.-O. Mewes, M. R. Andrews, N. J. van Druten, D. S. Durfee, D. M. Kurn, and W. Ketterle. Phys. Rev. Let., 75, 3969, 1995.

[15] T.D. Lee and C.N.Yang, Phys. Rev., 116, 25, 1959.

[16] T.D. Lee and C.N. Yang, Phys. Rev., 105, 119 (1975).

[17] T. J. Greytak and D. Kleppner. "Lectures on spin polarized hydrogen", in G. Gryndberg and R. Stora, editors, "Tendances actuelles en physique atomique". Elsevier, 1984. 
[18] I. F. Silvera and J. T. M. Walraven. "Spin polarized atomic hydrogen", in D. F. Brewer, editor, Progress in Low Temperature Physics. North Holland, Amsterdam, 1986.

[19] K. Huang, C.N. Yang and J.M. Luttinger, Phys. Rev., 105, 776 (1957)

[20] T.D. Lee and C.N.Yang, Phys. Rev., 113, 1165, 1959

[21] M. Abramowitz and I.A. Stegun. Handbook of Mathematical Functions, Dover, 1965.

[22] P. Grüter, thesis (Paris 1996).

[23] W. Kohn and J.M. Luttinger, Phys. Rev. Lett., 15, 524 (1965); J.M. Luttinger, Phys. Rev. 150, 202 (1966).

[24] M.Yu. Kagan and A.V. Chubukov, JETP Lett. 47, 614 (1988).

[25] P. Grüter and F. Laloë. J. de Physique I, 5, 1255, 1995.

[26] H. Stein, K. Moravec and G. Röpke, preprint 


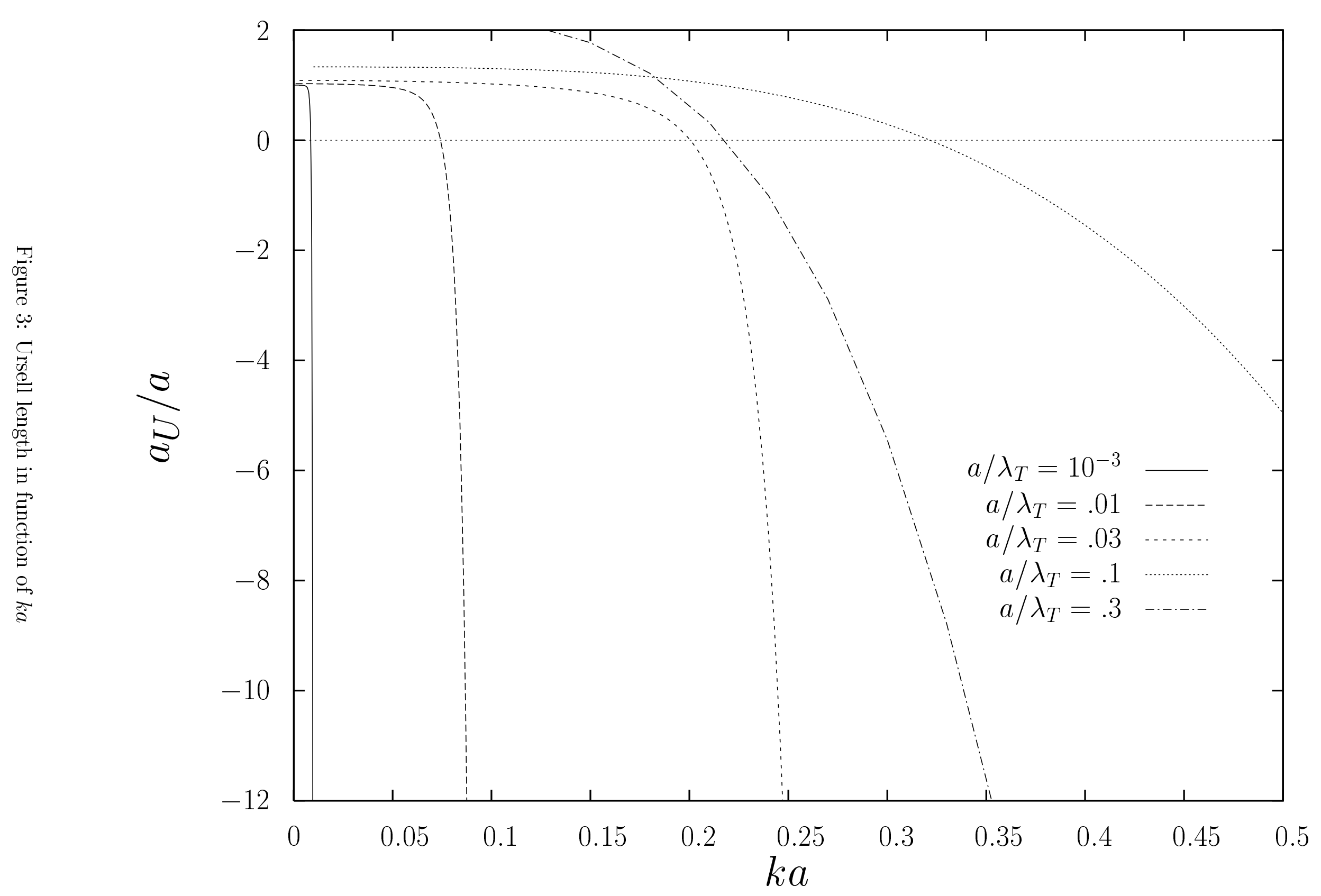




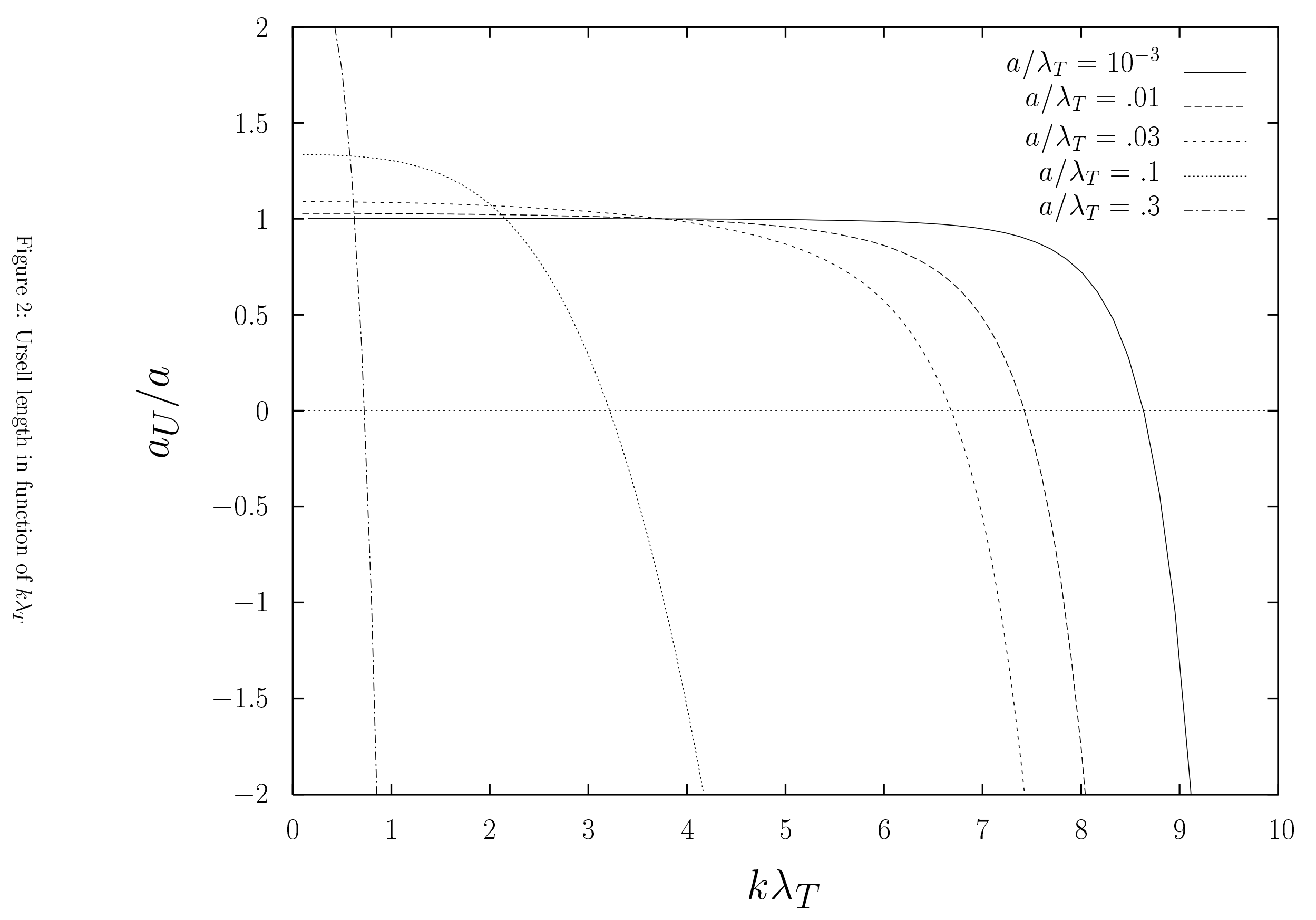




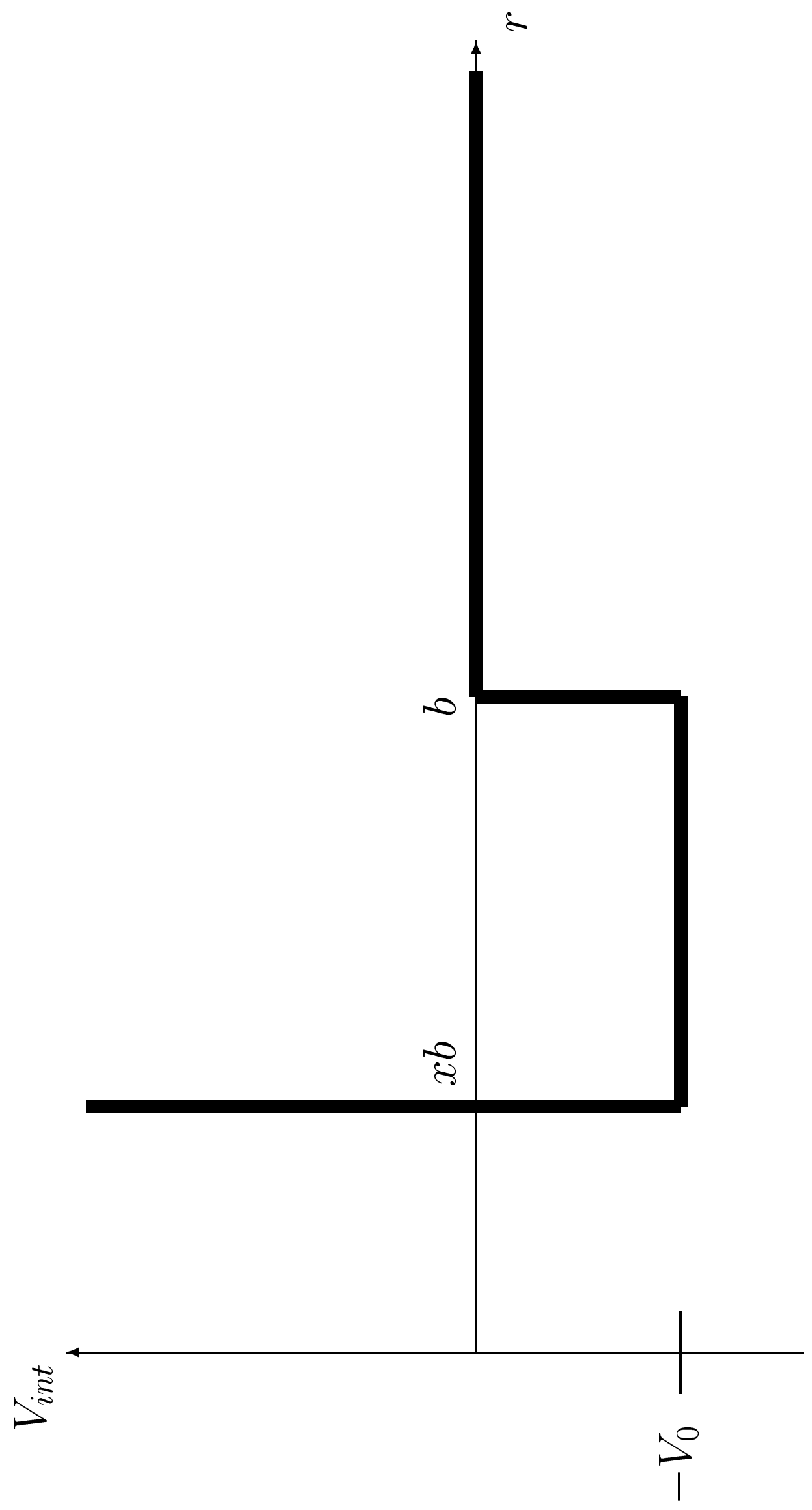

Figure 1: Potential used for the discussion in section 4.2 


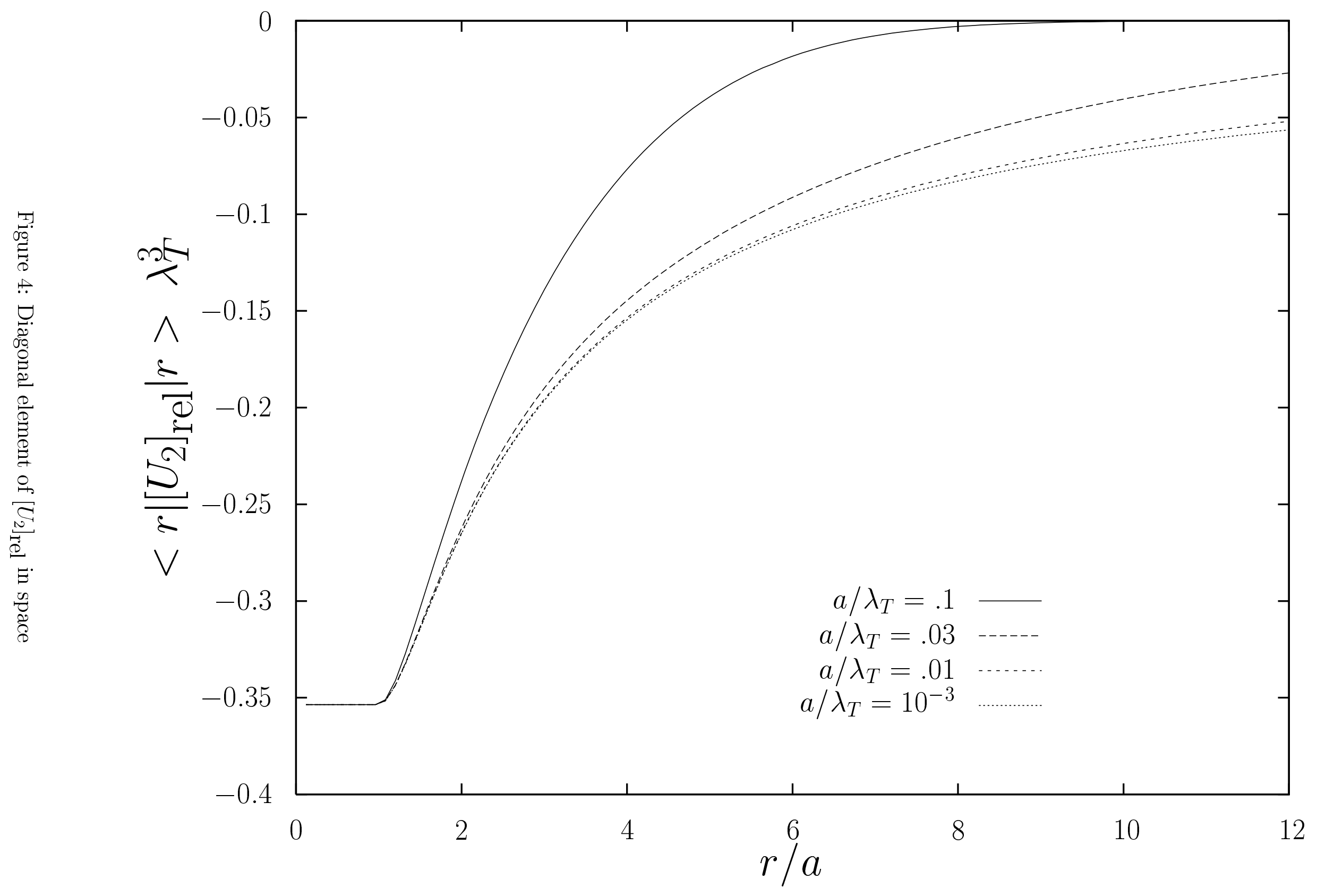

